\title{
Clinical and Polysomnographic Characteristics of Patients with Excessive Daytime Sleepiness
}

\author{
Yun Kyung Park, $\mathrm{MD}^{1}$, Kyung-Ha Noh, $\mathrm{MD}^{2}$, Seung Bong Hong, MD, $\mathrm{PhD}^{3}$, Eun Yeon Joo, MD, $\mathrm{PhD}^{3}$ \\ 'Department of Neurology, Bundang Jesaeng General Hospital, Seongnam, Korea \\ 2Department of Neurology, Good Gang-An Hospital, Busan, Korea \\ ${ }^{3}$ Department of Neurology, Neuroscience Center, Samsung Medical Center, Sungkyunkwan University School of Medicine, Seoul, Korea
}

Received: May 14, 2018

Revised: June 12, 2018

Accepted: June 26, 2018

\section{Correspondence}

Eun Yeon Joo, MD, PhD

Department of Neurology,

Neuroscience Center, Samsung Medical Center

Sungkyunkwan University School of Medicine,

81 Irwon-ro, Gangnam-gu, Seoul 06351, Korea

Tel +82-2-3410-3597

Fax +82-2-3410-0052

E-mail ejoo@skku.edu

ORCID

Yun Kyung Park

https://orcid.org/0000-0002-0725-1633

Kyung-Ha Noh

https://orcid.org/0000-0001-8349-2634

Seung Bong Hong

https://orcid.org/0000-0003-1684-637X

Eun Yeon Joo

https://orcid.org/0000-0003-1233-959X
Background and Objective Excessive daytime sleepiness (EDS) is a common symptom of many sleep disorders. EDS is the result of disturbed sleep or the sleep-waking process. The measurement of the degree of EDS is important for diagnosis and for its correlation with the severity of sleep disorders. We aimed to compare the characteristics of sleep disorders which were related to EDS and to investigate whether the Epworth Sleepiness Scale (ESS) and multiple sleep latency test (MSLT) were correlated with the polysomnographic parameters.

Methods 387 patients with EDS who completed both polysomnography (PSG) and MSLT were included. The severity of EDS was evaluated using ESS. Comparison of demographic, PSG, and MSLT results according to the final diagnosis and obstructive sleep apnea (OSA) subgroups categorized by the Apnea-Hypopnea Index.

Results The ESS score did not differ between the groups, except for narcolepsy type 1 and delayed sleep phase disorder $(\mathrm{p}=0.026)$. While the ESS score showed weak correlation with some PSG parameters, the mean sleep latency (mSL) of MSLT showed a significant and stronger correlation with PSG parameters. The mSL was significantly shorter in patients with severe OSA ( $4.6 \pm 3.0$, $\mathrm{p}<0.001)$ while the ESS did not show any difference among the different severities of OSA ( $\mathrm{p}=$ 0.754). Parameters which reflect OSA severity showed significant correlations with mSL.

Conclusions ESS is insufficient for reflecting differences in the types and severities of sleep disorders, so the objective parameters of PSG and MSLT are necessary for more precise diagnosis. There was significant but weak correlation with $\mathrm{mSL}$ in the case of ESS $\geq 10$, indicating that ESS was a poor screening tool but could be a convenient tool for follow-up study, rather than repeated MSLT.

Sleep Med Res 2018;9(1):32-38

Key Words Excessive daytime sleepiness, Epworth sleepiness scale, Multiple sleep latency test.

\section{INTRODUCTION}

Excessive daytime sleepiness (EDS) is one of the most frequent complaints in sleep laboratory visits [1], and one of the cardinal symptoms for the diagnosis of central disorders of hypersomnolence [2]. EDS is characterized by increased sleep pressure at daytime, commonly assumed to be the result of disturbed sleep [1] or deficient control of the sleep-waking process [3]. Therefore, measurement of the degree of EDS is important as certain degrees of EDS are required for the diagnosis of specific sleep disorders, and the degree of EDS is correlated with the severity of sleep disorders [4]. EDS can also be used to assess the therapeutic response of sleep disorders.

Among the various methods used for the measurement of EDS, the Epworth Sleepiness Scale (ESS) [5] and the multiple sleep latency test (MSLT) [6] are commonly used. ESS is useful to screen for subjective EDS, while MSLT measures objective EDS through standardized tests in the sleep laboratory. 
Previous studies have found conflicting results regarding whether EDS measured by ESS or MSLT correlates better to the severity of sleep disorders, especially to the severity of obstructive sleep apnea (OSA) [4]. Another study showed that ESS had a better ability to distinguish narcolepsy from normal subjects than MSLT [7].

The purpose of the present study was to assess the final diagnostic composition of sleep disorders which were related to EDS, and to investigate whether ESS and MSLT correlated with polysomnographic parameters.

\section{METHODS}

\section{Patient Selection}

From January 2008 to February 2012, we retrospectively reviewed treatment-naive patients who had completed both polysomnography (PSG) and MSLT in our tertiary neurological sleep laboratory. Six hundred thirty patients were initially included and 154 of them were excluded because of an incomplete dataset. Among the 476 eligible patients, 387 patients with ESS $\geq 10$ and a clear-cut final diagnosis were evaluated (Fig. 1). ESS $\geq 10$ is accepted to indicate EDS [5], and we excluded subjects with ESS $<10$ because the validity of ESS below cut-off value has not been proven. The final diagnosis was made according to the international classification of the sleep disorder-3 criteria [2], and medical records were reviewed and questionnaires were evaluated for accurate diagnosis.

The ethical approval of study was obtained by the Institutional Review Board of Samsung Medical Center (Study No. 2017-02-126). Written informed consent was not required because of the retrospective nature of the investigation.

\section{Sleep Studies}

PSG was conducted using an 18-channel recording system (Embla Co., Broomfield, CO, USA). We used six electroencephalogram channels, two electro-oculogram channels, a four channel electromyography for chin, intercostal muscles, and the right and left anterior tibialis, channels for nasal flow, arterial oxyhemoglobin saturation, and electrocardiography. PSG records were analyzed manually according to the criteria of the American Academy of Sleep Medicine [8].

MSLT was performed on the day after nocturnal PSG and consisted of five nap trials during $20 \mathrm{~min}$ at 2-h intervals across the day, beginning $1.5 \mathrm{~h}$ after waking up [6]. The mean sleep latency (mSL) (mean time to fall asleep) and number of cases of sleep-onset rapid eye movement (REM) sleep (REM sleep appearing within $15 \mathrm{~min}$ after sleep onset) were documented. The patients were classified as suffering from mild [Apnea-Hypopnea Index (AHI), $5 \leq \mathrm{AHI}<15 / \mathrm{h}]$, moderate $(15 \leq \mathrm{AHI}<$ $30 / \mathrm{h})$, severe $(30 \leq \mathrm{AHI}<50 / \mathrm{h})$, or very severe $(\mathrm{AHI} \geq 50 / \mathrm{h})$.

\section{Clinical Measurement}

Prior to sleep tests, all patients completed questionnaires that included the ESS, Stanford Sleepiness Scale (SSS), and Beck Depression Inventory (BDI). According to the ESS questionnaire, an ESS $\geq 10$ was defined as sleepy and ESS $<10$ as non-sleepy. The SSS evaluates the current level of subjective sleepiness ranging from 1 (alert) to 7 (sleep onset soon) [9]. BDI is a tool for measuring the severity of depressive symptoms (BDI $\geq 10$, clinically significant) [10].

\section{Statistical Analysis}

Continuous variables are summarized with means and standard deviations and compared using analysis of variance and Tukey's post hoc analysis. Categorical variables are summarized using frequencies and percentages and compared among clusters using a chi-square test. p-values of $<0.05$ were considered to be statistically significant. Correlation analysis was performed using Pearson's correlation coefficients. The analysis was performed using SPSS 17.0 (SPSS Inc., Chicago, IL, USA).

\section{RESULTS}

\section{Patient Characteristics}

Subjective EDS as assessed by ESS scores $\geq 10$ was present in 387 (81.3\%) of 476 patients. Fig. 1 showed each group's (with ESS $\geq 10$ vs ESS $<10$ ) composition by the categorized sleep disorder diagnosis. There was no significant difference in the composition of the diagnosis between the two groups $(\mathrm{p}=0.150)$. Two hundred forty-five (63.3\%) of the 387 patients were male, with the mean age upon evaluation being $40.6 \pm 18.9$ (ranging from 7 to 79) years. The detailed diagnosis of subjective EDS group includes OSA (28\%), narcolepsy type 1 (N1) (16.8\%), narcolepsy 2 (N2) (15.5\%), idiopathic hypersomnia (IH) (9.6\%), restless leg syndrome (RLS) (6.2\%), chronic insomnia disorder (5.9\%), and delayed sleep phase disorder (DSPD) (5.7\%). Cases with other diseases were very few (insufficient sleep, long sleeper, REM sleep behavior disorder, etc).

Of the chronic insomnia disorder patients, $82.6 \%$ had comorbid conditions, which included depression $(n=2)$, mild OSA $(n=10)$, delayed sleep-wake phase disorder $(n=3)$ and others.

\section{Clinical and Sleep Study Characteristics of Subjective EDS Patients}

338 subjects with seven more frequent sleep disorders were further analyzed (Table 1). RLS or OSA patients who showed ESS $\geq 10$ were older than narcolepsy and IH patients with ESS $\geq$ $10(\mathrm{p}<0.001)$. The proportion of male patients was highest in OSA $(\mathrm{p}<0.001)$ and Body Mass Index was higher in the OSA and $\mathrm{N} 1$ groups $(\mathrm{p}<0.001)$.

The ESS score did not differ between groups except for N1 and DSPD $(\mathrm{p}=0.026)$. OSA showed the shortest total sleep 


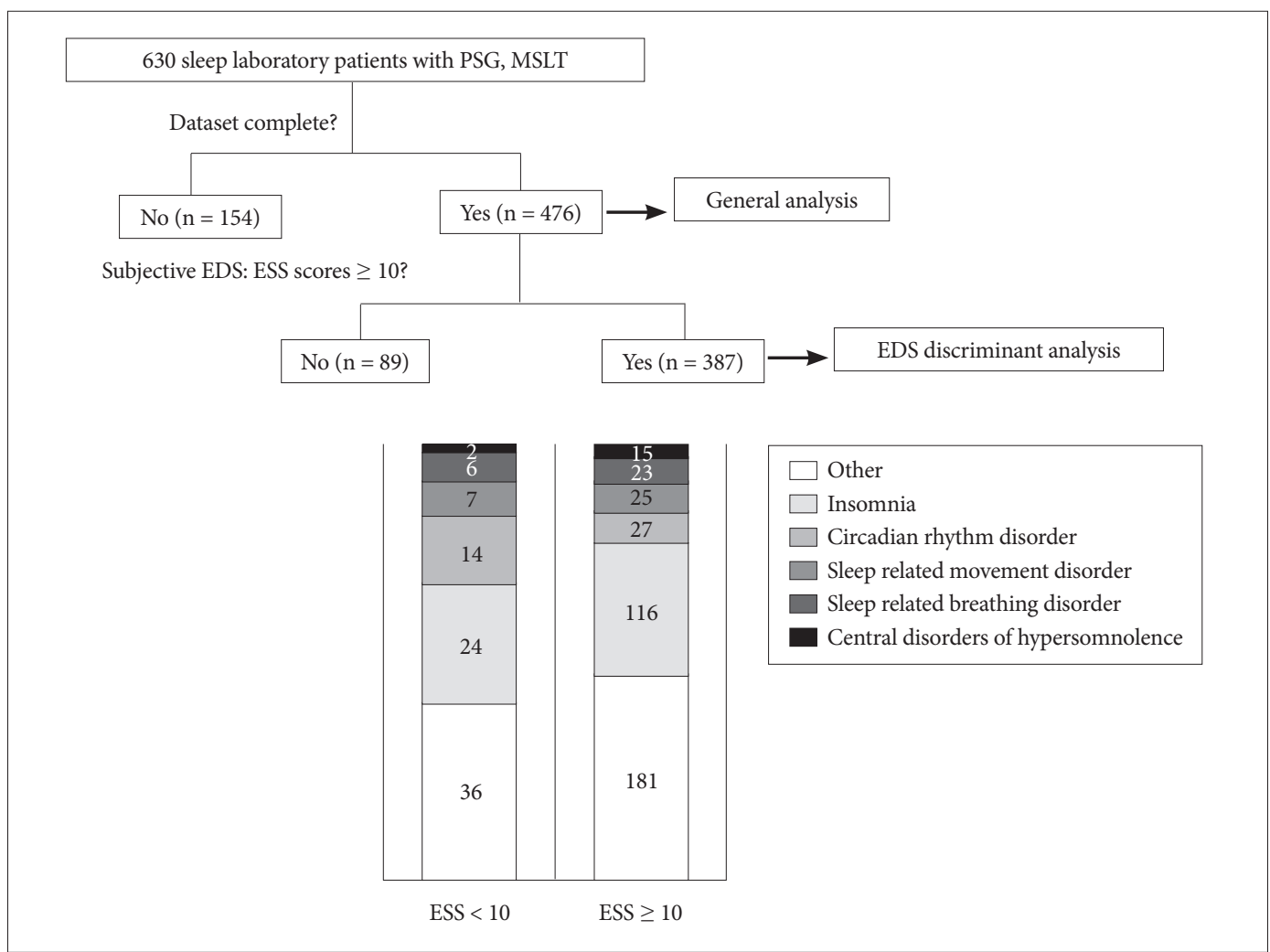

Fig. 1. Flow diagram depicting the inclusion procedure in this retrospective study. Central disorders of hypersomnolence includes narcolepsy type 1 and 2, idiopathic hypersomnia, hypersomnia due to medication, insufficient sleep syndrome and long sleeper. Sleep related breathing disorder: obstructive sleep apnea and catathrenia. Sleep related movement disorder: restless leg syndrome and periodic limb movement disorder. Circadian rhythm disorder: delayed sleep-wake phase disorder, advanced sleep-wake phase disorder, irregular sleepwake rhythm disorder and shift work disorder. Insomnia: chronic insomnia disorder. Other (ESS < 10): normal $1+$ sleep related epilepsy 1. Other (ESS $\geq 10$ ): normal 7 + REM sleep behavior disorder 4 + sleep talking 1 + sleep related epilepsy 3 . ESS: Epworth Sleepiness Scale, REM: rapid eye movement, PSG: polysomnography, MSLT: multiple sleep latency test, EDS: excessive daytime sleepiness.

time (TST), highest Arousal Index (AI), longest wakefulness after sleep onset (WASO), and lowest sleep efficiency (SE) $(\mathrm{p}<$ 0.001 ) compared to the other groups. N1 showed significantly shorter sleep latency (SL) and REM latency than other sleep disorders on PSG $(\mathrm{p}<0.001)$.

MSLT results revealed significant differences among the sleep disorders. The mSL was shortest in N1 and N2, and longest in insomnia and DSPD $(p<0.001)$. Sleep onset REM was more frequent in N1 and N2 than in other sleep disorders ( $\mathrm{p}<0.001)$.

In correlation analysis, ESS showed correlation with SSS, BDI, WASO, and mSL $[\mathrm{r}=-0.139(\mathrm{p}=0.011), \mathrm{r}=0.135(\mathrm{p}=0.015)$, $\mathrm{r}=0.131(\mathrm{p}=0.016)$, and $\mathrm{r}=-0.123(\mathrm{p}=0.024)$, respectively], and $\mathrm{mSL}$ showed correlation with SL, SE, and WASO $[\mathrm{r}=0.408$ $(\mathrm{p}<0.001), \mathrm{r}=-0.211(\mathrm{p}<0.001)$, and $\mathrm{r}=0.186(\mathrm{p}<0.001)$, respectively].

\section{Comparing Patients with Different Severity of OSA}

Of the 107 OSA patients with ESS $\geq 10,20$ (18.7\%), 37 (34.6\%), $20(18.7 \%)$, and $30(28.0 \%)$ were classified as mild, moderate, severe, and very severe OSA, respectively. The ESS, MSLT, and PSG parameters of the four groups of different severity of OSA are shown in Table 2. No significant differences in the TST, SE, and SL were found between the groups. No differences in the ESS score were found, but mSL was significantly shorter in the moderate to very severe groups than it was in the mild group $(p<0.001)$. Patient with very severe degrees of OSA were more likely have lower levels of lowest oxyhemoglobin saturation $\left(\mathrm{SaO}_{2}\right)$, longer lenghts of longest apneic duration, and lesser slow wave sleep (SWS).

The correlations between the sleep parameters, the mSL of MSLT, and ESS score are shown in Table 3. ESS score was not correlated with any of the sleep parameters investigated. $\mathrm{mSL}$ showed negative correlation with $\mathrm{AHI}$, longest apneic duration, and $\mathrm{AI}$, and positive correlation with lowest $\mathrm{SaO}_{2}$ and amount of SWS.

\section{Comparison of Sleep Disorders according to EDS Severity}

Fig. 2 compares sleep disorders in the three groups according to the ESS severity $\left(10^{-14}, 15-19,20-24\right)$ and $\mathrm{mSL}$ degree of MSLT $(\mathrm{mSL} \leq 8,8<\mathrm{mSL} \leq 15,15<\mathrm{mSL})$.

There was no significant difference in ESS severity between the sleep disorders. $(p=0.157)$. However, each sleep disorder showed significant differences in terms of mSL severity $(\mathrm{p}<0.001)$. 
Park YK, et al

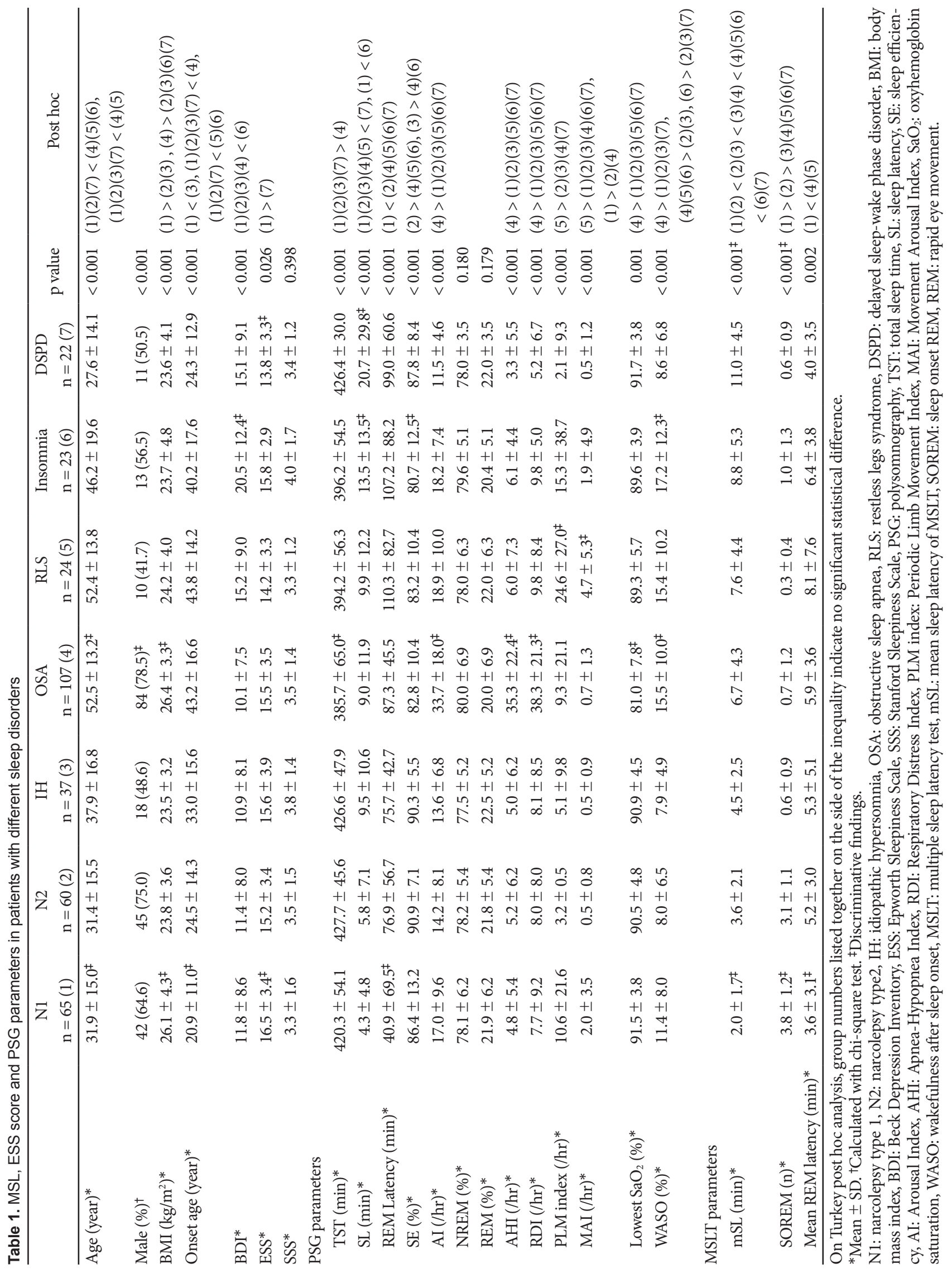


Table 2. Comparison of ESS, MSL and PSG parameters by different severity of OSA

\begin{tabular}{|c|c|c|c|c|c|c|}
\hline & $\begin{array}{c}\text { Mild } \\
5 \leq \mathrm{AHI}<15 \\
\mathrm{n}=20(1)\end{array}$ & $\begin{array}{c}\text { Moderate } \\
15 \leq \mathrm{AHI}<30 \\
\mathrm{n}=37(2)\end{array}$ & $\begin{array}{c}\text { Severe } \\
30 \leq \mathrm{AHI}<50 \\
\mathrm{n}=20(3)\end{array}$ & $\begin{array}{c}\text { Very severe } \\
50 \leq \mathrm{AHI} \\
\mathrm{n}=30(4)\end{array}$ & $\mathrm{p}$ value & Post hoc \\
\hline ESS & $15.5 \pm 3.3$ & $15.8 \pm 4.0$ & $15.9 \pm 3.3$ & $14.9 \pm 3.0$ & 0.754 & \\
\hline SSS & $3.6 \pm 1.6$ & $3.7 \pm 1.5$ & $3.3 \pm 1.6$ & $3.4 \pm 1.3$ & 0.750 & \\
\hline $\operatorname{mSL}(\min )$ & $10.2 \pm 4.4$ & $7.0 \pm 4.3$ & $5.9 \pm 3.8$ & $4.6 \pm 3.0^{*}$ & $<0.001$ & $(1)>(2)(3)(4)$ \\
\hline $\mathrm{AI}(/ \mathrm{hr})$ & $18.9 \pm 7.5$ & $22.8 \pm 8.2$ & $38.1 \pm 12.2$ & $54.1 \pm 15.2^{*}$ & $<0.001$ & $(1)(2)<(3)<(4)$ \\
\hline TST (min) & $407.8 \pm 42.4$ & $393.7 \pm 76.0$ & $364.4 \pm 58.8$ & $375.4 \pm 63.2$ & 0.122 & \\
\hline SE (\%) & $83.0 \pm 8.9$ & $84.1 \pm 10.5$ & $80.1 \pm 11.3$ & $82.8 \pm 10.7$ & 0.579 & \\
\hline $\mathrm{SL}(\min )$ & $12.6 \pm 16.4$ & $7.0 \pm 6.3$ & $7.4 \pm 7.1$ & $10.0 \pm 15.5$ & 0.329 & \\
\hline Lowest $\mathrm{SaO}_{2}(\%)$ & $86.4 \pm 4.6$ & $83.7 \pm 5.1$ & $79.0 \pm 8.2$ & $75.5 \pm 8.2^{*}$ & $<0.001$ & $(1)>(3)(4),(2)>(4)$ \\
\hline Longest apneic durations (sec) & $25.6 \pm 16.9$ & $39.4 \pm 20.7$ & $47.7 \pm 23.8$ & $64.3 \pm 30.8^{*}$ & $<0.001$ & $(1)<(3)(4),(2)<(4)$ \\
\hline Slow wave sleep (\%) & $7.0 \pm 7.7$ & $3.2 \pm 5.0$ & $2.8 \pm 3.9$ & $2.1 \pm 3.6^{*}$ & 0.008 & $(1)>(2)(4)$ \\
\hline REM sleep (\%) & $21.3 \pm 4.2$ & $21.5 \pm 5.3$ & $19.6 \pm 6.6$ & $17.7 \pm 9.4$ & 0.121 & \\
\hline
\end{tabular}

Data are presented as mean \pm standard deviation.

* Discriminative findings.

ESS: Epworth Sleepiness Scale, SSS: Stanford Sleepiness Scale, mSL: mean sleep latency of MSLT, AI: Arousal Index, TST: total sleep time, SE: sleep efficiency, SL: sleep latency, PSG: polysomnography, OSA: obstructive sleep apnea, $\mathrm{SaO}_{2}$ : oxyhemoglobin saturation, REM: rapid eye movement.
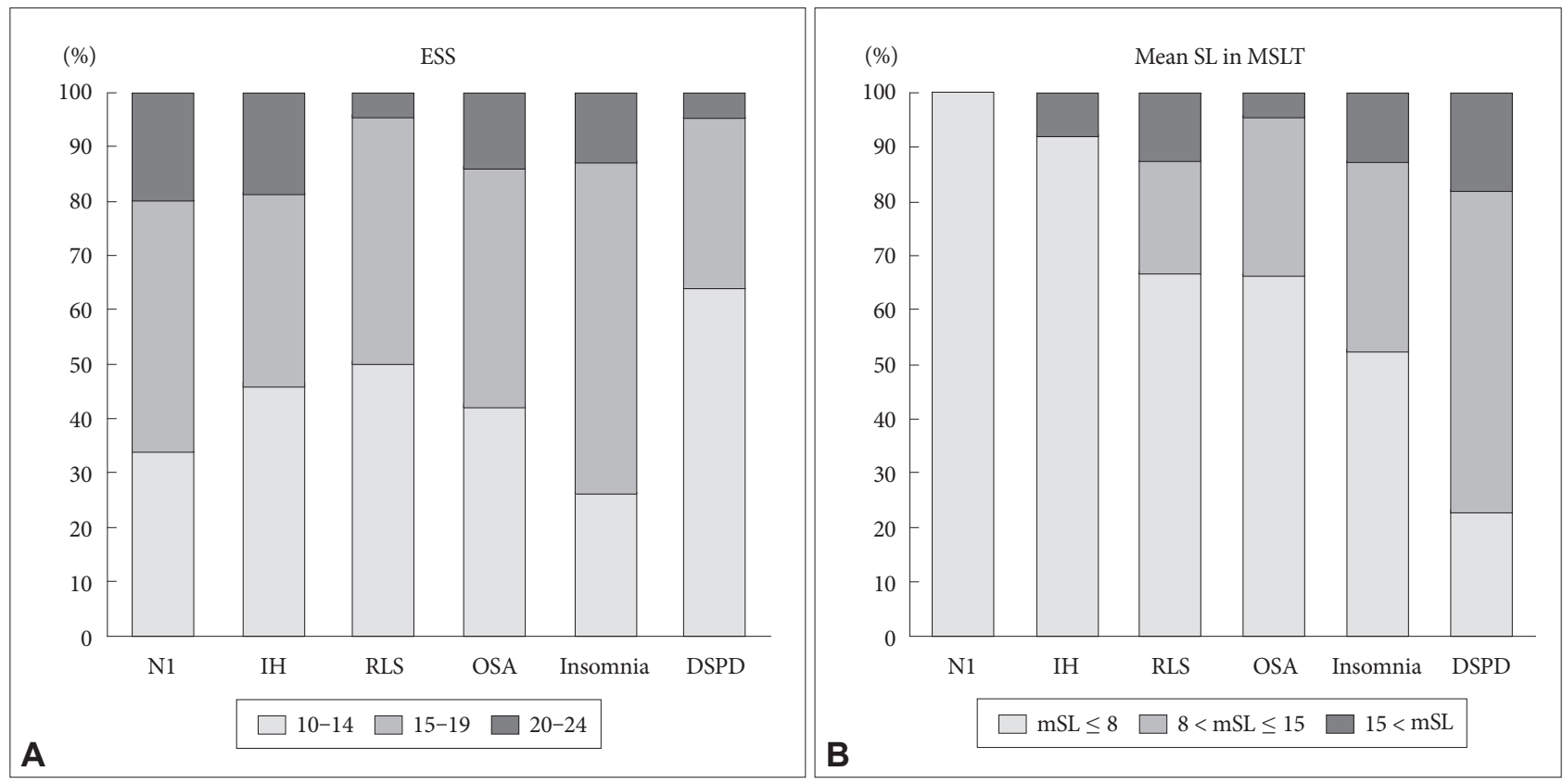

Fig. 2. Histogram showing distribution of patient with specific diagnosis in each ESS score range (A) and in each $\mathrm{mSL}$ range (B). No significant difference in proportion of ESS score among sleep disorders $(p=0.192)$, but mean sleep latency showed significant difference among sleep disorders $(\mathrm{p}=0.007)^{*}$ by Pearson's chi-square test. ${ }^{*} \mathrm{~N} 1$ and $\mathrm{IH}$ cases are excluded from analysis of mean SL comparison, because short Mean SL is one of diagnostic criteria of narcolepsy and idiopathic hypersomnia. ESS: Epworth Sleepiness Scale, MSLT: multiple sleep latency test, N1: narcolepsy type 1, IH: idiopathic hypersomnia, RLS: restless legs syndrome, OSA: obstructive sleep apnea, DSPD: delayed sleep-wake phase disorder.

\section{DISCUSSION}

In the present study, we investigated the characteristics of sleep disorders which contribute to EDS in patients. Central disorders of hypersomnia, sleep-related breathing disorders, sleeprelated movement disorders, chronic insomnia disorder and, circadian rhythm disorder were the major etiologies of EDS in our subjects, which were comparable to those found by a previous study [11]. Mean ESS score and ESS score distribution appeared to be similar among sleep disorders, which means ESS is not a discriminative test for the diagnosis of sleep disorders.

While ESS scores were significant but showed a weak corre- 
Table 3. Pearson correlation coefficient $(r)$ between $\mathrm{mSL}$, ESS score and different PSG parameters measured

\begin{tabular}{|c|c|c|c|c|}
\hline & \multicolumn{2}{|c|}{ ESS } & \multicolumn{2}{|c|}{$\mathrm{mSL}$} \\
\hline & $\mathrm{r}$ & $\mathrm{p}$ & $\mathrm{r}$ & $\mathrm{p}$ \\
\hline AHI & -0.118 & 0.227 & -0.392 & $<0.001$ \\
\hline Lowest $\mathrm{SaO}_{2}$ & 0.100 & 0.305 & 0.302 & 0.002 \\
\hline Longest apneic duration & -0.104 & 0.286 & -0.241 & 0.012 \\
\hline Amount of slow wave sleep (\%) & -0.093 & 0.342 & 0.376 & $<0.001$ \\
\hline $\mathrm{AI}$ & -0.047 & 0.529 & -0.349 & $<0.001$ \\
\hline
\end{tabular}

ESS: Epworth Sleepiness Scale, SSS: Stanford Sleepiness Scale, mSL: mean sleep latency of MSLT, PSG: polysomnography, AHI: Apnea-Hypopnea Index, $\mathrm{SaO}_{2}$ : oxyhemoglobin saturation, $\mathrm{AI}$ : Arousal Index.

lation with mSL and some of the PSG parameters, $\mathrm{mSL}$ of MSLT showed significant and stronger correlations with PSG parameters. The correlation between MSL of MSLT and PSG parameters become more evident in OSA subgroup analysis. The mSL was significantly shorter in patients with severe OSA, while the ESS score did not significantly differ amongst patients with different severities of OSA. PSG parameters which reflect OSA severity, such as $\mathrm{AHI}$, lowest $\mathrm{SaO}_{2}$, longest apneic duration, amount of SWS, and AI showed significant correlation with $\mathrm{mSL}$. This suggests that $\mathrm{mSL}$ of MSLT might be a better indicator than ESS of EDS severity with reference to the severity of OSA.

Previous studies of sleep perception and MSLT in chronic insomnia disorder showed lower ESS scores $(2.81 \pm 3.48)$ and higher $\mathrm{mSL}(14.05 \pm 4.4 \mathrm{~min})$ in patients [12]. In contrast to the previous study, we found EDS patients with insomnia and they showed high ESS scores $(15.8 \pm 2.9)$ and relatively high $\mathrm{mSL}$ of MSLT ( $8.8 \pm 5.3 \mathrm{~min})$. High ESS score seemed contradictory to the hyperarousal tendency of insomnia. The reasons for the discrepancy between the previous study and the present study are the high BDI score $(20.5 \pm 12.4)$ which is known to be attributable to the condition of EDS [13] and having comorbid condition in insomnia subjects in our study.

The discrepancy in ESS and MSLT have been discussed in previous studies $[14,15]$ and similar findings were replicated in our study. The difference in ESS and MSLT might reflect different aspects of EDS. The nature of MSLT is how quickly the subject falls asleep in a specific situation. The mSL of MSLT is measured objectively, while it may vary with the time of day and in response to a sleep-deprived state [7]. The ESS provides subjects' average sleep propensity in daily life. It may be strongly affected by gender, psychological factors, and the subjective perception of fatigue [16,17].

We should address some limitation of this study. Subjects in this study may not represent the general population. This study demonstrated the current states of patients with EDS who visited the sleep clinic in the university-affiliated hospital. We analyzed the data of patients who completed both PSG and MSLT during a certain period. MSLT is a diagnostic test for central hypersomnia thus usually performed for patients in whom central hypersomnia is suspected. This could be a reason that central hypersomnia accounts for some large portions. The preference of morningness and eveningness might affect EDS [18], which we did not account for in this study. Considering that previous studies reported no overall differences in EDS across chronotypes, the effects of chronotype would be minimal [19].

This study presented that ESS was not sensitive enough to discriminate sleep disorders or to reflect the severity of OSA. On the other hand, ESS may be a convenient study of EDS in some circumstances, such as in a follow-up study for the evaluation of treatment response instead of MSLT, considering the correlation between ESS $\geq 10$ and mSL of MSLT.

We suggest that ESS is unreliable for screening, but objective parameters of PSG and MSLT might be needed for final assessments.

\section{Acknowledgments}

This research was supported by Basic Science Research Program through the National Research Foundation of Korea funded by the Ministry of Science, ICT \& Future Planning, Republic of Korea (2017R1A2B4003120) and by Samsung Biomedical Research Institute grant (SMX1170571).

\section{Conflicts of Interest}

The authors have no financial conflicts of interest.

\section{Authors' Contribution}

Conceptualization: Park YK, Noh KH, Joo EY. Data curation: Park YK, Noh KH. Formal analysis: Park YK, Noh KH. Funding acquisition: Joo EY. Methodology: Park YK, Noh KH, Joo EY. Resources: Joo EY, Hong SB. Supervision: Joo EY, Hong SB. Validation: Park YK, Noh KH, Joo EY. Writing_original draft: Park YK, Noh KH. Writing_review \& editing: Park YK, Joo EY.

\section{REFERENCES}

1. Roehrs T, Carskadon MA, Dement WC, Roth T. Daytime sleepiness and alertness. In: Kryger MH, Roth T, Dement WC. Principles and Practice of Sleep Medicine. Philadelphia: Saunders 2000;43-52.

2. Medicine AAoS. International classification of sleep disorders. 3rd ed. Darien, IL: American Academy of Sleep Medicine 2014.

3. Naumann A, Daum I. Narcolepsy: pathophysiology and neuropsychological changes. Behav Neurol 2003;14:89-98.

4. Fong SY, Ho CK, Wing YK. Comparing MSLT and ESS in the measurement of excessive daytime sleepiness in obstructive sleep apnoea syndrome. J Psychosom Res 2005;58:55-60.

5. Johns MW. A new method for measuring daytime sleepiness: the Ep- 
worth sleepiness scale. Sleep 1991;14:540-5.

6. Littner MR, Kushida C, Wise M, Davila DG, Morgenthaler T, Lee-Chiong T, et al. Practice parameters for clinical use of the multiple sleep latency test and the maintenance of wakefulness test. Sleep 2005;28:113-21.

7. Johns MW. Sensitivity and specificity of the multiple sleep latency test (MSLT), the maintenance of wakefulness test and the epworth sleepiness scale: failure of the MSLT as a gold standard. J Sleep Res 2000;9:5-11.

8. Iber C A-IS, Chesson AL, Quan SF. The AASM manual for the scoring of sleep and associated events: rules, terminology, and technical specifications. Westchester, IL: American Academy of Sleep Medicine 2007.

9. Hoddes E, Dement W, Zarcone V. The development and use of the Stanford sleepiness scale (SSS). Psychophysiology 1972;9:150.

10. Beck A, Steer RA, Brown GK. Beck Depression Inventory-II. 2nd ed. San Antonio, TX: Psychological Corporation 1996.

11. Kretzschmar U, Werth E, Sturzenegger C, Khatami R, Bassetti CL, Baumann CR. Which diagnostic findings in disorders with excessive daytime sleepiness are really helpful? A retrospective study. J Sleep Res 2016;25:307-13.

12. Huang L, Zhou J, Li Z, Lei F, Tang X. Sleep perception and the multiple sleep latency test in patients with primary insomnia. J Sleep Res 2012; 21:684-92.
13. Bixler EO, Vgontzas AN, Lin HM, Calhoun SL, Vela-Bueno A, Kales A. Excessive daytime sleepiness in a general population sample: the role of sleep apnea, age, obesity, diabetes, and depression. J Clin Endocrinol Metab 2005;90:4510-5.

14. Chung KF. Use of the epworth sleepiness scale in chinese patients with obstructive sleep apnea and normal hospital employees. J Psychosom Res 2000;49:367-72.

15. Olson LG, Cole MF, Ambrogetti A. Correlations among epworth sleepiness scale scores, multiple sleep latency tests and psychological symptoms. J Sleep Res 1998;7:248-53.

16. Chervin RD, Aldrich MS. The epworth sleepiness scale may not reflect objective measures of sleepiness or sleep apnea. Neurology 1999;52: 125-31.

17. Chervin RD. Sleepiness, fatigue, tiredness, and lack of energy in obstructive sleep apnea. Chest 2000;118:372-9.

18. Taillard J, Philip P, Bioulac B. Morningness/eveningness and the need for sleep. J Sleep Res 1999;8:291-5.

19. Rosenthal L, Day R, Gerhardstein R, Meixner R, Roth T, Guido P, et al. Sleepiness/alertness among healthy evening and morning type individuals. Sleep Med 2001;2:243-8. 